\title{
A MIXED SPACEBORNE SENSOR APPROACH FOR SURFACE MODELING OF AN URBAN SCENE
}

\section{ABSTRACT}

3D surface models are vital for sustainable urban management studies and there is a nearly unlimited range of possible applications. Along- or across-track pairs from the same set of sensor imagery may not always be available or uneconomic for a certain study area. Therefore a photogrammetric approach is proposed in which a Digital Surface Model (DSM) is extracted from a stereo pair of satellite images, acquired by different sensors. The results demonstrate that a mixed sensor approach may offer a sound alternative to the more established alongtrack pairs. However, one should consider several criteria when selecting a suitable stereo pair. Two cloud-free acquisitions are selected from the Ikonos and Quickbird image archive, characterized by sufficient overlap and optimal stereo constellation in terms of complementarity of azimuth and elevation angle. A densely built-up area in Istanbul, Turkey, covering $151 \mathrm{~km}^{2}$ and with an elevation ranging between sea level and approximately $160 \mathrm{~m}$ is presented as test site. In addition to the general complexity of modeling the surface and elevation of an urban environment, multi-sensor image fusion has other particular difficulties. As the images are acquired from a different orbital pass, at a different date or instant of time and by a different sensor system, radiometric and geometric dissimilarities can occur which may hamper the image matching process. Strategies are presented for radiometric and geometric normalization of the multi-temporal and multi-sensor imagery and to deal with the differences in sensor characteristics. The accuracy of the generated surface model is assessed by comparison with 3D reference points, 3D rooftop vector data and surface models extracted from an along-track Ikonos stereo pair and an Ikonos triplet. Results show that it is feasible to extract a DSM of a highly urbanized area from a mixed sensor pair, with accuracies comparable with those observed from a DSM 
extracted from an along-track pair. Hence, the flexibility of reconstructing valuable elevation models is greatly increased by considering the mixed sensor approach. 


\subsection{INTRODUCTION}

Digital surface models have become an important data source for geospatial analysis, with a wide range of DSM-derived products able to be generated for various applications. As such, DSMs can be applied in areas such as monitoring, urban and environmental planning, risk and disaster management, telecommunication planning, flood analysis and microclimate research among others.

3D terrain descriptions can be generated by various measurement techniques, such as airborne laserscanning, Interferometric Synthetic Aperture Radar (InSAR), photogrammetry with automatic image matching algorithms or manual digitization. In the presented work, a photogrammetric approach is proposed for the extraction of a DSM from a multi-sensor stereo pair, i.e. a stereo pair consisting of two satellite images acquired by different very high resolution Earth observation sensors.

According to photogrammetric theory, stereoscopic images cover the same part of the Earth's surface, but are acquired from a slightly different viewpoint. Image matching techniques detect conjugate points on both images. Once two image projections of the same terrain point are matched, disparity or height parallax can be measured and the elevation of the particular point calculated.

To obtain a stereo pair from High Resolution Satellite Imagery (HRSI), two constellation types may be employed. In the case of along-track or in-track stereoscopy, a forward and a backward image are acquired nearly simultaneously on the same orbital pass of the satellite. In the case of acrosstrack stereoscopy, images cover the same geographical location, but are acquired from two different orbital passes of a satellite, by rolling the sensor sideways. Although along-track stereo produces undoubtedly more reliable and robust results due to the reduced radiometric and geometric discrepancies between images, across-track stereo, consisting of images from the same sensor or different sensors, is extremely valuable for various reasons. Certain shortcomings of along-track acquisition have stimulated the interest in acrosstrack acquisition schemes:

(i) Along-track stereo pairs are scarce, as sensor rotation in stereo imaging prohibits the possibility of taking other scenes within between. Thus for 
satellite companies it is not economic to generate stereo pairs (Buyuksalih \& Jacobsen, 2007);

(ii) If along-track is available for a given study area, acquisition must be programmed and therefore the data can be quite expensive to acquire. Not to mention the fact that it can take quite some time to acquire cloud-free imagery;

(iii) Not all pushbroom sensor systems have the capacity to acquire in-track pairs.

However, there is an increasing amount of Very High Resolution (VHR) space sensors systematically acquiring monoscopic scenes. The latter can be combined, if they capture the same area from a different viewing angle. A distinction can be made between across-track pairs, made up of images from the same sensor, and across-track pairs comprised of images from different sensors. As it can be difficult to find a suitable pair within the image archive of a single sensor, a multisensor approach increases the flexibility of 3D model reconstruction from satellite archive data and facilitates subsequent applications. Despite the potential of mixed sensor stereo pairs, different sensors have different modalities and characteristics. Also a large time interval can exist between the two image acquisitions. Both of these factors may cause image projections of the same geographical location to have different radiometric or geometric properties. This results in surface (in)dependent grey value differences, which hamper the image matching process.

A number of interesting studies can be found in the literature which discusses the issue of DSM extraction from along-track pairs and across-track HRSI pairs of the first type. Zhang \& Gruen (2006) reported a Root Mean Square value for height differences (RMSZ) of $3.38 \mathrm{~m}$ and a bias of $0.55 \mathrm{~m}$ for a DSM extracted over an urban area, based on an Ikonos along-track triplet and 39 Ground Control Points (GCPs). In Poon et al. (2005), a DSM extracted from an along-track Ikonos pair, covering an urban area, was assessed with a resulting RMSZ value of $4 \mathrm{~m}$ and a bias of $1.5 \mathrm{~m}$. Devriendt et al. (2006) measured an RMSZ of $11.91 \mathrm{~m}$ and a bias of $1.84 \mathrm{~m}$ in the analysis of a very dense urban city core. The DSM was derived from an along-track Ikonos pair. In all these studies Light Detection and Ranging (LiDAR) data was used as reference. In Zhang et al. (2002) the accuracy of an Ikonos across-track pair covering a mountainous rural area was assessed 
by its comparison with a digital terrain model derived from 1:5000 contour maps. The pair had a convergent angle of $17.4^{\circ}$ and a small time gap of 12 days. An RMSZ value of $3.96 \mathrm{~m}$ and a bias of $1.3 \mathrm{~m}$ were reported.

Relatively few studies have been reported regarding the application of across-track stereo pairs, constructed from mixed sensor imagery. Ikonos and Quickbird images are often used synergetically to generate time series for Land Use / Land Cover (LULC) change studies. However, only few have studied the geometric conditions required for accurate surface model and ortho-image generation from mixed sensor imagery. Multi-sensor DSM generation experiments were first described and assessed in Welch et al. (1990), Raggam \& Almer (1991) and Toutin (1998). Mentioned studies discuss DSM extraction from a stereo pair that is comprised of a SPOT and Landsat image. Due to the coarse resolution of these sensors, sufficient terrain height differences must be present in order to yield observable disparities.

During the last decade, across-track stereoscopy has received renewed interest because of the development of VHR sensors. Li et al. (2007) assessed the 3D geopositioning accuracy of different possible combinations of Ikonos and Quickbird images. However, only the geometric accuracy of the image triangulation (relative and absolute image orientation process) was addressed, with image matching of mixed sensor imagery and DSM extraction not investigated. Raggam (2006) reported interesting results, concerning acrosstrack image matching, with a variety of different datasets and case studies investigated, including photogrammetric fusion of Ikonos and Quickbird imagery. However the DSMs involved were not extracted over urban terrain, but over Mediterranean forest areas, while a reference model with the required accuracy and level of detail was not available.

In this work, an approach is presented which aims to extract a DSM over an urbanized area from an across-track stereo pair, consisting of a forward Ikonos image and a backward Quickbird acquisition. The three-dimensional information is extracted according to a highly automated matching algorithm. Objectives are threefold:

(i) Implementation of a feasible methodology to extract height information from multi-sensor HRSI, according to machine-based photogrammetric matching. 
(ii) As not every combination of two monoscopic images from the archive constitutes a good stereo pair, the posited approach will attempt to provide a brief overview of the criteria that should be taken into account for the selection of suitable, complementary imagery.

(iii) The necessity of geometric and radiometric normalization of multitemporal, multi-sensor imagery is emphasized, to compensate for the differences between the images of the non-conventional mixed sensor pair.

The research presented here is conducted within the framework of the MAMUD project (Measuring And Modeling of Urban Dynamics) funded by the Belgian Science Policy STEREO (Support to The Exploitation and Research of Earth Observation data) program. In section two, the characteristics and constellation of the mixed sensor stereo pair are discussed, with a focus on the criteria for the selection and fusion of VHR spaceborne imagery. Section three discusses the surface model generation approach and consists of four subsections. Subsection one deals with the radiometric preprocessing of the images and discusses the necessity of applying locally adaptive contrast enhancement and normalization. Subsection two reviews geometric sensor models, a procedure necessary in order to establish the relationship between image space and object space. Subsection three highlights the necessity of geometric normalization of the imagery, in terms of epipolar resampling. Subsection four discusses the preferred image matching strategy and setting of the most relevant parameters. Besides, the DSM is interpolated and the results discussed. In section four, DSM extraction accuracy and surface model quality are assessed and reported by means of qualitative and quantitative analysis. The paper concludes with a brief summary and discussion of the methodology and the obtained results.

\subsection{MiXed SENSOR IMAGE DATASET \& STUDYFIELD}

\subsubsection{Image dataset}

Both the Ikonos and Quickbird platform are equipped with a pushbroom sensor. In pushbroom geometry, an image is typically acquired dynamically line by line via projection on an array of sensors. While the platform is moving, the sensor 
sweeps over the terrain and scans image strips consecutively at different instants in time, with a very narrow angle of view. For each line, a different set of values exists for the six exterior orientation parameters. A holistic image is dynamically acquired by the joining of successive strips.

An image acquired by a pushbroom sensor actually encloses two geometric projections: a central or perspective projection along the Charged-Coupled Device (CCD) ruler perpendicular to the satellite motion direction, and a parallel projection in the direction of flight (Wolniewicz \& Ke, 2006). Such complex image geometry demands more sophisticated processing techniques than traditional frame camera geometry. The main image characteristics of Ikonos and Quickbird acquisition are listed in table 5-1.

Table 5-1. Parameters of multi-sensor, across-track stereo imagery.

\begin{tabular}{lcc}
\cline { 2 - 3 } & Ikonos GEO & $\begin{array}{c}\text { Quickbird Standard } \\
\text { 2A }\end{array}$ \\
\hline Acquisition date & $2005-05-16$ & $2002-03-07$ \\
Scan direction & Forward & Backward \\
Spatial resolution & $1 \mathrm{~m}$ & $0.61 \mathrm{~m}$ \\
Elevation angle & $80.9^{\circ}$ & $76.2^{\circ}$ \\
Collection azimuth & $23.5^{\circ}$ & $119^{\circ}$ \\
Sun elevation & $65.5^{\circ}$ & $40.3^{\circ}$ \\
Sun azimuth & $148.4^{\circ}$ & $153.2^{\circ}$ \\
Cloud cover & 0 & 0 \\
\hline
\end{tabular}

\subsubsection{Selection criteria for suitable images}

The most important criteria for the selection of suitable images, to constitute a mixed sensor stereo pair, are a minimal time interval between acquisition dates, comparable spatial resolution, cloud-free acquisition and complementary stereo constellation. Below, the different criteria are discussed more in detail.

In table 5-1, a large temporal discrepancy between acquisition dates, including a seasonal shift, is apparent. The different atmospheric and illumination conditions and sensor characteristics produced by this discrepancy result in surface-independent grey value variations for projections of the same terrain. In addition, surface-dependent changes such as new construction works and changes in the phenological state of vegetation will occur, severely impeding 
image matching. Despite of this temporal constraint, the selected images can be considered the most optimal candidates for the area under study in terms of the other criteria.

Studies of urban environments require a certain metric quality and level of image detail for the accurate identification and modeling of individual terrain features such as buildings. At the time of the launch of the MAMUD project, Ikonos and Quickbird were the only commercial Earth observation sensors yielding (sub-)meter spatial resolution in panchromatic mode. Furthermore, the resolution of images constituting a mixed sensor pair must be of comparable size. Otherwise two different-looking sets of pixels as well as a divergence in spectral information will be produced for a particular terrain feature.

The preferred image products here are raw acquisitions, geometrically corrected by the image provider for systematic distortions due to the sensor, the platform and the Earth rotation and curvature (Toutin, 2001). Both images are cloud-free acquisitions. Minimum cloud coverage is an important criterion as clouds and their shadows hamper the image matching process.

Perhaps the most important criterion which candidate images should satisfy is that they project the Region Of Interest (ROI) from complementary viewing angles. Constituting so-called 'stereo constellation', satellite azimuth and elevation angles have a fundamental influence on the acquisition accuracy, and thus the extent to which height information can be extracted from the stereo model. Stereo constellation or stereo acquisition geometry can be determined by the convergence angle or its equivalent base-to-height ratio. The convergence angle is an angular parameter measuring the geometric relationship between two rays through projections of a common object point.

In traditional analogue photogrammetry, where elevation is measured manually by an operator, the $\mathrm{B} / \mathrm{H}$ ratio serves as the main indicator to predict the geometric accuracy of the model. The larger the $\mathrm{B} / \mathrm{H}$ ratio, the greater the range of depth perception and the better the vertical mensuration accuracy (Cain, 1989). However in the case of automatic 3D extraction from spaceborne imagery, many other components affect the accuracy such as the terrain type, image distortions, the applied matching algorithms, selected GCPs and the sensor model employed.

The relationship between the convergence angle and satellite azimuth and elevation angles is illustrated in figure 5-1 and expressed as in equation (5-1): 


$$
\cos \delta=\sin \alpha_{1} \sin \alpha_{2}+\cos \alpha_{1} \cos \alpha_{2} \cos \left(\theta_{2}-\theta_{1}\right)
$$

where $\delta$ is the stereo pair convergence angle; $\alpha_{i}(i=1,2)$ are the satellite elevation angles; and $\theta_{i}(i=1,2)$ the satellite azimuth angles. According to equation (5-1), the Ikonos-Quickbird stereo pair has a convergence angle of $17.2^{\circ}$ or a corresponding base-to-height ratio of 0.4 , values that should result in rather small image disparities. From a theoretical point of view, a strong stereo constellation should be in the order of $\mathrm{B} / \mathrm{H}=1$ to yield large height parallax. However, the designed base-to-height ratio for satellite systems with fixed stereo capabilities differs significantly. The $\mathrm{B} / \mathrm{H}$ ratio of PRISM on ALOS is set to 1.0 (forward view + backward view). In the case of ASTER on Terra it is set to 0.6 and for OPS/VNIR on JERS-1, 0.3 .

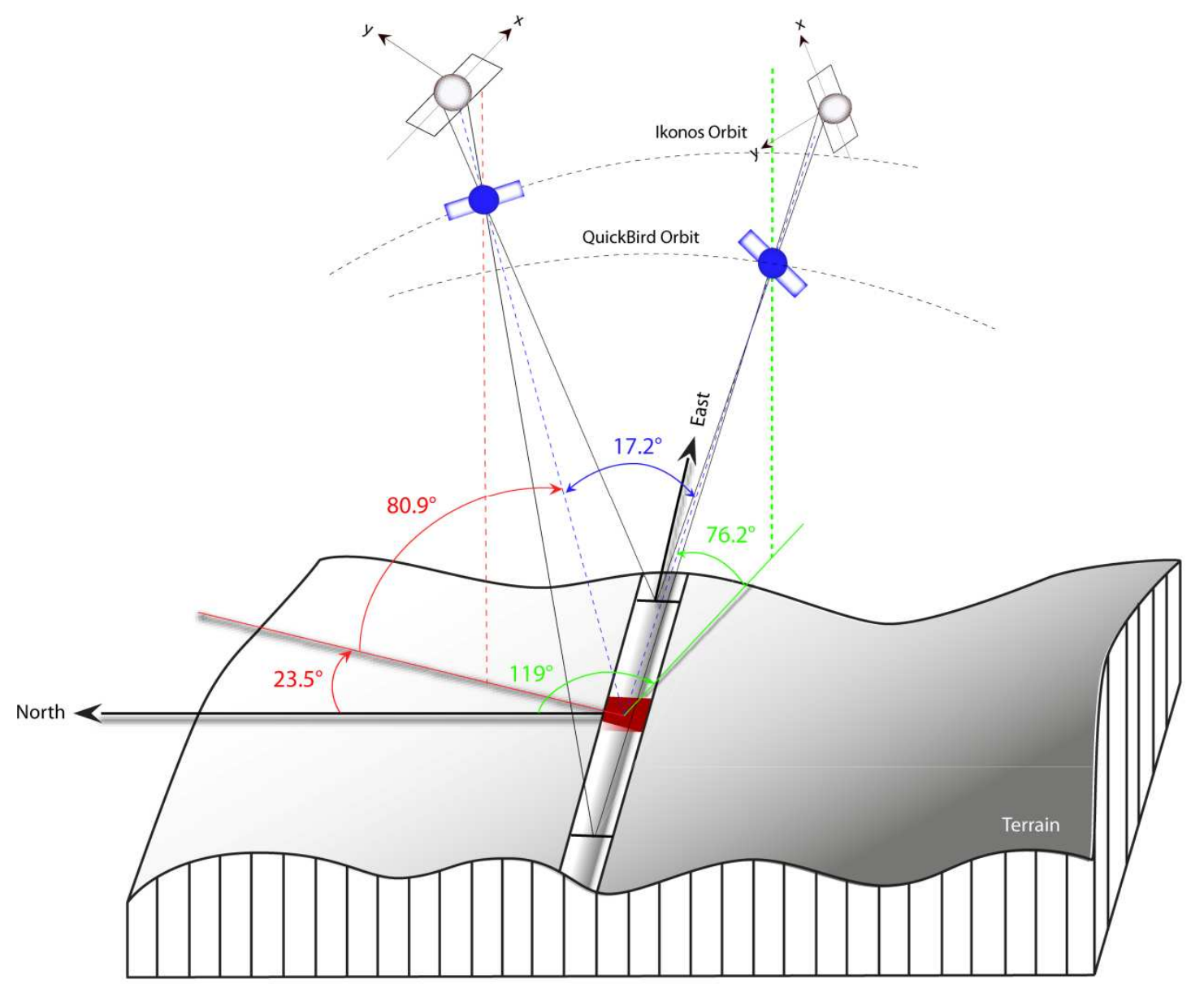

Figure 5-1. Ikonos-Quickbird stereo pair constellation with the convergence angle annotated in blue. 
In Li et al. (2007), the 3D geopositioning accuracy of different possible combinations of one Ikonos and one Quickbird along-track stereo pair is assessed, by comparison with a set of 3D check points. From their experimental results, the mixed sensor pairs consisting of an Ikonos and a Quickbird acquisition yield a Root Mean Square Error (RMSE) for X, Y and Z in the same range as that for the along-track pairs. The authors conclude that it is mainly satellite stereo geometry, which plays the more significant role in triangulation accuracy, and not the type of imagery: the bigger the convergence angle, the better the extracted elevation accuracies as the stereo exaggeration factor or observed disparity becomes larger. However in Li et al. (2007), only the geometric accuracy of image triangulation is addressed, with image matching of mixed sensor imagery and (accuracy of) DSM extraction not investigated. Jacobsen (2006) and Buyuksalih \& Jacobsen (2007) confirm that height accuracy is linear depending upon the base-to-height ratio in case of:

(i) manual 3D point acquisition by a human operator;

(ii) automatic height measurement by an image matching algorithm in open terrain or flat areas.

However, this is not the case for automatic height measurement of a dense urban environment. In Jacobsen (2006) and Buyuksalih \& Jacobsen (2007) the influence of the convergence angle is assessed for DSM extraction from different VHR stereo pairs. The presented experimental results illustrate that a smaller convergence angle has major advantages in urbanized areas, by reducing image dissimilarities, building displacement and occluded areas, and allowing a stereo view of the street surface.

The test site investigated in this paper is a densely urbanized area. Hence, to reduce occlusion and image dissimilarities resulting from building displacement, a stereo constellation with a small convergence angle is preferred, despite the loss of accuracy for height parallax measurements. This elucidates the choice of near-Nadir elevation angles.

\subsubsection{Test site}

A part of the megacity of Istanbul, Turkey is chosen as a test site. Despite its size, the city is very compact and is concentrated along the Bosphorus strait. The 
research area consists of the corresponding coverage of Ikonos and Quickbird images (see figure 5-2). It concerns a densely built-up environment with an area of approximately $151 \mathrm{~km}^{2}$. The area contains Istanbul's urban core, its historic peninsula and extends to the urban fringe in the North. Geomorphologically the terrain is characterized by a rugged topography with elevation ranging from sea level up to $162 \mathrm{~m}$. Most building types within the study area are irregularly constructed and connected multi-family townhouses and apartment buildings. As a result building size and shape vary markedly.

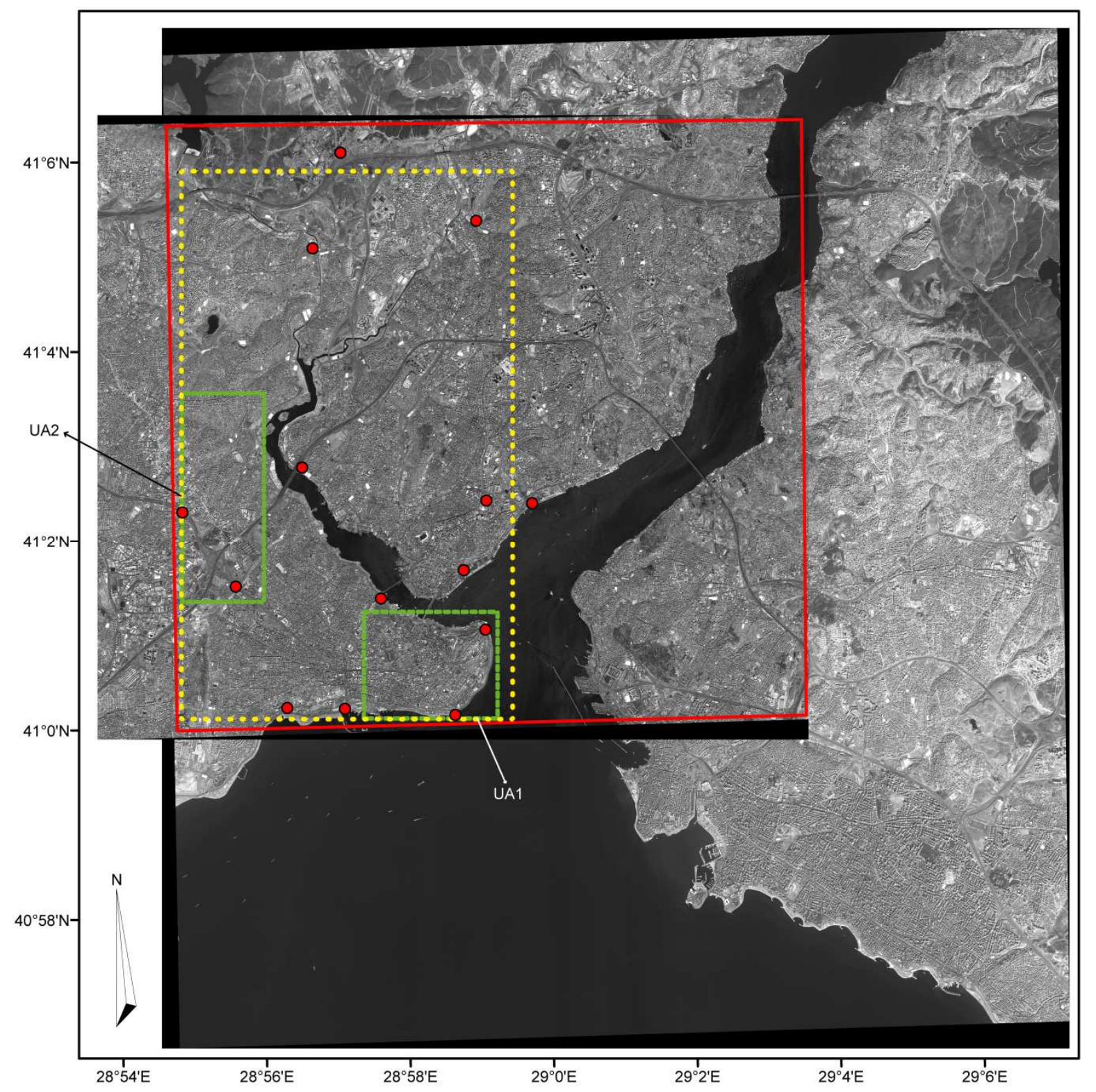

Figure 5-2. Overview map with (i) study area, delineated by red polygon; (ii) yellow dotted and green dashed polygons respectively indicating the DSM area extracted from an Ikonos triplet and a 3D building vector dataset. Both 
are used as references for the accuracy assessment; (iii) red dots illustrating the used GCPs to refine the RPC sensor model (overlap of Quickbird PAN image, 2002-03-07 and Ikonos PAN image, 2005-05-16).

\subsection{SURFACE MODEL GENERATION}

The successive steps of the applied methodology for extraction of a surface model from the mixed sensor pair are discussed in following subsections (see figure 5-3).

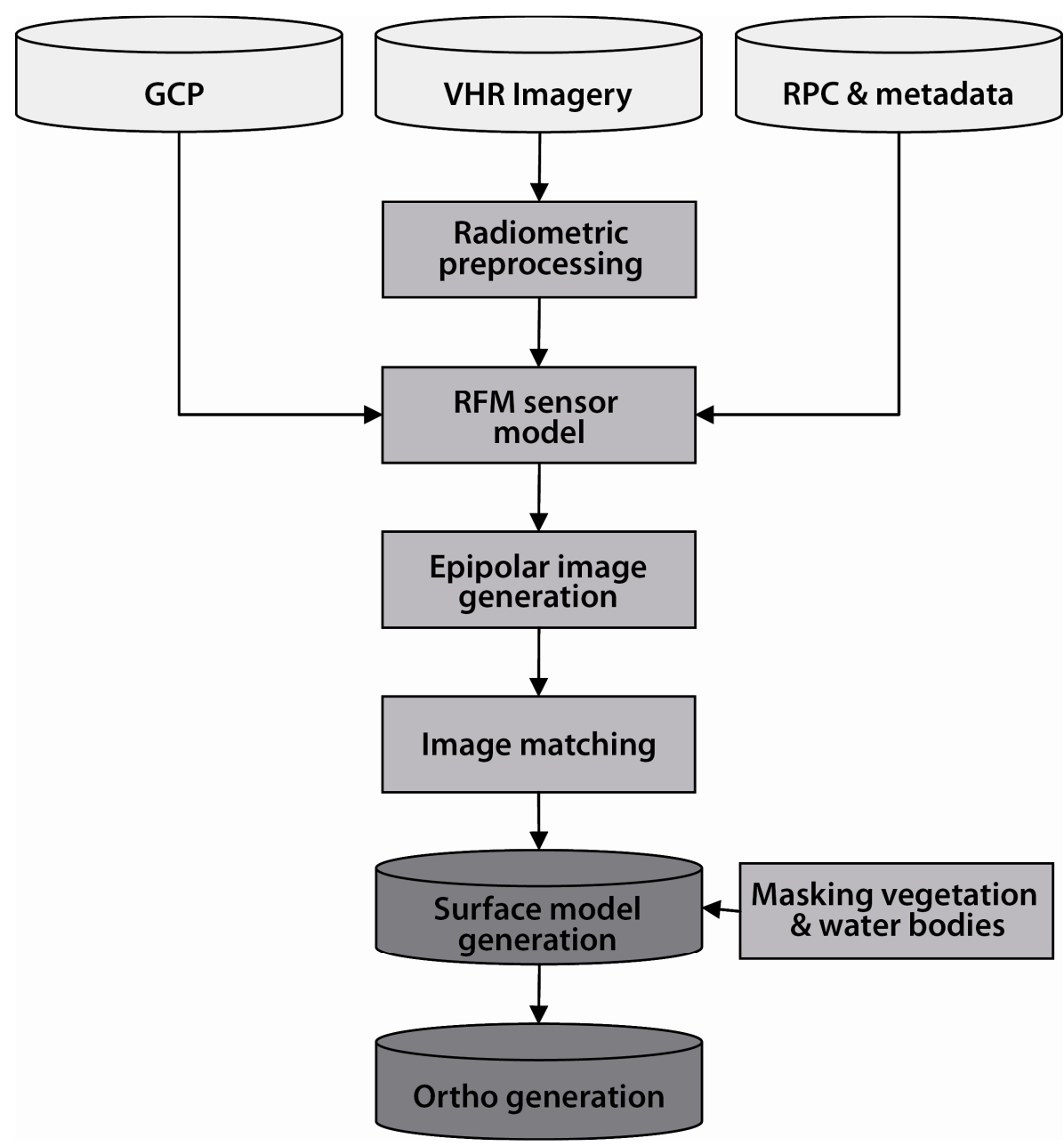

Figure 5-3. Extraction of a DSM from a mixed sensor pair flowchart.

\subsubsection{Radiometric preprocessing}

Images of a mixed sensor pair are acquired from different orbital passes, on different dates at different instants in time and by different sensor systems. As a result, the two projections of a given terrain point can have a divergent radiometric response. This hampers the matching of corresponding terrain 
features. Radiometric dissimilarities in imagery also occur in along-track pairs, as the images are acquired from slightly different orbital positions. However these effects are much smaller.

Surface-independent grey value variation resulting from discrepancies in illumination, atmospheric conditions and sensor modalities is a fundamental problem, but can be greatly reduced by the application of contrast enhancement and normalization. Furthermore, radiometric enhancement improves (weak) texture patterns in areas with a small dynamic range in grey level, such as those exhibiting shadowing or oversaturation. However, radiometric preprocessing cannot cope with terrain-dependent dissimilarities, such as changes in the phenological state of vegetation, land cover change or demolished / new structures.

Two major categories of radiometric enhancement can be distinguished. Linear Global Contrast Enhancement (GCE) performs an overall intensity scaling, usually leading to excessive lighting conditions in the brightest portions of the image and vice versa to poor lighting of the darkest portions. Non-linear Local Contrast Enhancement (LCE) mainly enhances the visibility of local details in an image (Al-Amri et al., 2010).

In order to enhance the overall contrast of each individual image and to normalize the radiometric differences between the multi-temporal imagery, here a Wallis filter was preferred and applied (Wallis, 1976). The Wallis filter performs a non-linear, locally adaptive contrast enhancement, taking the following general form:

$$
g^{w}(x, y)=g(x, y) r_{1}+r_{0}
$$

with

$$
\begin{gathered}
r_{1}=\frac{c s_{h}}{c s_{g}+\left(\frac{s_{h}}{c}\right)} \\
r_{0}=b m_{h}+\left(1-b-r_{1}\right) m_{g}
\end{gathered}
$$

where $r_{0}$ and $r_{1}$ are respectively the additive and multiplicative parameter; $g^{w}(x, y)$ and $g(x, y)$ the filtered and original image; $m_{g}$ and $s_{g}$ the original mean and 
standard deviation values; $m_{h}$ and $s_{h}$ the target mean and standard deviation values; and $c$ and $b$ the contrast expansion and brightness forcing constants.

As the filter performs a spatially-varying enhancement of the image, it can provide good local contrast at both ends of the dynamic range of grey values. Actually, a large kernel partitions the image into different 21 by 21 pixel regions, and within each region the local contrast is optimized based on local histogram information. The window size of 21 pixels is chosen as it represents an optimal compromise between a small window that will enhance mainly tiny and unimportant features and a large window that will result in significant loss of detail.

The mean and standard deviation of radiometric variation is computed for each partition in the original image. The target mean and standard deviation are user-specified and set at 128 and 64 respectively in the case of 8-bit imagery. A higher mean value will brighten the image, while a higher target standard deviation will yield a bigger contrast stretch. The contrast expansion constant $c$ and brightness forcing constant $b$ are set to 0.75 and 0.6 , respectively. The Wallis-filtered image is a weighted combination of the original mean and standard deviation values and the target mean and standard deviation. The weight parameter is determined by the brightness forcing constant, which can have a value between zero and one. A weight value of 0.6 will calculate an image with $60 \%$ target values and $40 \%$ original values (Jazayeri \& Fraser, 2008). The contrast expansion constant is a general image contrast enhancement function between zero and one that determines the amount of stretch over the dynamic range. Despite the locally adaptive Wallis filter performing a global image normalization, this action cannot be categorized as an image normalization method s.s., since the radiometric properties of a given slave image are not adjusted to match a given reference image (Hall et al., 1991).

The effect of the Wallis filter when applied to an HRSI image is illustrated in figure 5-4. Figures 5-4(a) and 5-4(b) represent respectively an extract of the original and the Wallis-filtered Ikonos image, with the related histogram provided for both. The images show an area of tall buildings. In the original image very little contrast can be observed within the shadow areas, resulting in poor and erroneous image matching. A richer contrast can be detected in the Wallis-filtered image, with the radiometric filter locally enhancing existing texture patterns leading to optimization of contrast in the shadow areas. 

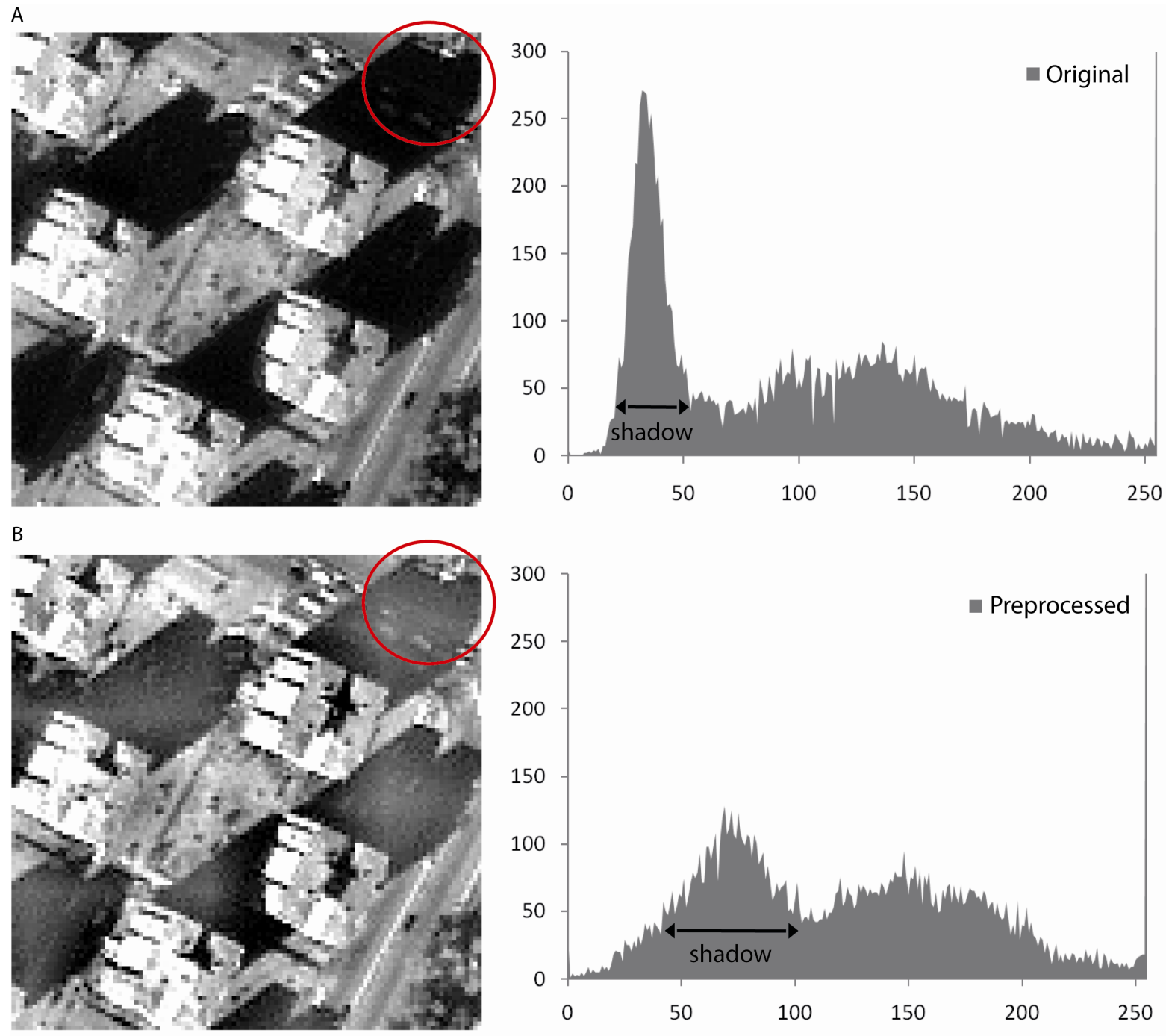

Figure 5-4(a) \& 5-4(b). Subscene of Ikonos image (Ikonos PAN, 2005-05-16), respectively before and after radiometric enhancement with related histogram.

The histograms presented in figure 5-4 describe the grey value frequency distribution. Both exhibit a bi-modal distribution, distinguishing shadow from normally-exposed areas. Pixel intensity values show a sharp high peak for the shadows in the original image. The small tonal range of the cluster indicates low contrast. However, a good overall distribution with a wide tonal range in the midtones can be observed. The mean value and standard deviation are 104 and 69 , respectively. The preprocessed image tends to have higher frequencies at the ends. The shadow peak is transformed into a more contrast-rich distribution, tending to the midtones and with a wider dynamic range. This transformation represents a gain in image information and detail. An important nuance that 
should be noted is that radiometric enhancement and normalization can only reduce - not eliminate - surface-independent grey value variation, and only enhance - not solve - weak texture patterns in improving image matching.

\subsubsection{Geometric sensor model}

To define the geometric properties of a sensor or to establish the relationship between 3D terrain points and projected image pixels, a geometric sensor model must be applied. Two types can be distinguished: physical / rigorous models and empirical models. Rigorous models represent the physical imaging process based on sensor geometry and system parameters, while empirical models are mathematical approximations. Rigorous sensor models are relatively simple and well-known from their use with perspective frame cameras. However, this is not the case with pushbroom sensors. Crespi et al. (2009) thoroughly discussed rigorous models for pushbroom sensors and reported that their complexity can be attributed to the fact that pushbroom sensors build an image dynamically from many individual lines, each acquired with proper position and attitude values. Because of this, physical-geometric 3D reconstruction from 2D imagery based on collinearity equations is not a straightforward task for pushbroom geometry.

The ultimate prerequisite for multi-sensor image fusion and DSM extraction is algorithmic fusion via the integration of the respective sensor models (Raggam et al., 1992). Because of the complexity of physical pushbroom sensor models and because of the need for an environment able to fuse mixed sensor imagery in the same triangulation, the Rational Functional Model (RFM) was preferred here. An RFM or Rational Polynomial Coefficient (RPC) model is an empirical sensor model which describes orientation information in terms of ratios of polynomial functions of object coordinates (Grodecki, 2001; Grodecki \& Dial, 2003):

$$
\begin{aligned}
& x=\frac{P_{1}(X, Y, Z)}{P_{2}(X, Y, Z)} \\
& y=\frac{P_{3}(X, Y, Z)}{P_{4}(X, Y, Z)}
\end{aligned}
$$


where $x$ and $y$ are the pixel coordinates expressed as image samples and lines. The third-order, 20-term polynomial is a function of latitude, longitude and elevation and takes the form:

$$
\begin{aligned}
P_{i}(X, Y, Z) & =a_{1}+a_{2} X+a_{3} Y+a_{4} Z+a_{5} X Y+a_{6} X Z+a_{7} Y Z+a_{8} X^{2}+a_{9} Y^{2} \\
& +a_{10} Z^{2}+a_{11} X Y Z+a_{12} X^{3}+a_{13} Y^{2}+a_{14} X Z^{2}+a_{15} X^{2} Y \\
& +a_{16} Y^{3}+a_{17} Y Z^{2}+a_{18} X^{2} Z+a_{19} Y^{2} Z+a_{20} Z^{3}
\end{aligned}
$$

where $\mathrm{X}, \mathrm{Y}, \mathrm{Z}$ are the object coordinates and $a_{i}(i=1-20)$ the rational polynomial coefficients. At present, RPCs are available for most HRSI and can be provided by the vendor for each image.

RFMs have the vital property of being platform-independent, with the same set of equations able to be applied directly to different images, even if they are acquired by different sensors (Shaker, 2008). Although the RFM sensor model has great potential, including its simplicity and independence from sensor geometry and platform, the model suffers from biases in sensor exterior orientation. This is because RPCs are solely derived from the satellite ephemeris and satellite attitude angles calculated by an on-board GPS / INS system. The terrain-independency of the model causes systematic errors. In the RFM model, the link between 2D image and 3D object space is described by a simple mathematical relation that does not consider the geometric-physical process of image projection.

As part of the methodology's aim for high geometric accuracy, systematic errors were reduced and the RPC sensor model further refined through the use of ground control information. A first order correction was applied to the model, based on a set of GCPs. GCPs were measured with C-NAV Differential GPS equipment. The system provides sub-10 cm horizontal accuracy and sub-20 cm vertical accuracy. GCP measurements at open spaces were pursued, although this was not straightforward within the dense urban core. Corners of sidewalks and grass-patches, surrounded by areas of contrasting color, were mainly selected. Most points are localized at major road intersections and near the shoreline due to the presence of more open spaces at these locations. In order to avoid extrapolation in planimetry and elevation, we aimed to achieve at a homogeneous distribution of points. However, only the western part of the test 
site is covered due to logistical constraints (see figure 5-2). Therefore only the western part will be compared with reference data in the accuracy assessment.

Rozycki \& Wolniewicz (2007) thoroughly investigated the influence of the number of used GCPs on DSM accuracy for Ikonos and Cartosat-1 imagery, concluding that 10 to 15 well-distributed GCPs need to be used to yield results within the theoretical accuracy range. Using a larger number of GCPs did not bring forth significant accuracy improvements, but instead increased the risk of adding an erroneously measured or badly pointed GCP, pulling the overall performance of the model down. Crespi et al. (2009) concluded the same for Quickbird imagery from their experimental results.

In total, 14 unambiguously identifiable points on both images with known map coordinates were used to refine the RFM model. The a priori geometric accuracy of the stereo model has an overall RMSE value of $0.81 \mathrm{~m}$ for $\mathrm{X}$ residuals, $0.87 \mathrm{~m}$ for $\mathrm{Y}$ residuals and $2.43 \mathrm{~m}$ for $\mathrm{Z}$ residuals. Once the scenes are triangulated according to an iterative least-squares stereo-bundle adjustment, the image orientation is established. This means that the three position and three orientation parameters per image as well as the relationship between 2D image and 3D object space are defined and additional image data processing can be performed.

\subsubsection{Epipolar resampling}

As the images are acquired from different orbital passes and sensor systems, geometric normalization, mainly required to remove shifts in the y-direction and differences in scale, rotation and spatial resolution, is vital for multi-sensor stereo mapping purposes. An epipolar resampling is therefore performed, involving the application of a relative registration between the images using a linear pixel transformation. Based on the determined orientation parameters, the images are then resampled to the same pixel size as that of the Ikonos image and rectified to an epipolar format. An affine transformation is applied that aligns the images via scaling, rotation and translation in Y-direction, thus enabling stereo vision and enhancing the subsequent image matching.

This preparatory normalization results in the same geometric properties being held by both stereo images, except for displacement caused by terrain topography (Raggam et al., 1992). Normalized images are generated, with 
conjugate points located approximately along the same image row or same horizontal epipolar line. The rectification process leaves the $x$-parallax or 'elevation induced pixel offset' between conjugate points unresolved. The $x$ parallax is linearly proportional to the depth of the corresponding object point across the air base connecting the involved perspective centers (Morgan et al., 2006).

In addition to geometric normalization, epipolar resampling boosts the matching algorithm as it constrains the search space and reduces matching ambiguities. The process of detecting homologous points is basically a twodimensional search algorithm but can be constrained to a one-dimensional search along epipolar line segments. An area-based matching algorithm is based on the calculation of correlation between patches on a given reference and search image. As the process requires images of the same resolution, the sampling rate of the Quickbird image is decreased to the $1 \mathrm{~m}$ resolution of the Ikonos acquisition. Down-sampling according to a nearest neighbor algorithm was then applied, in order to avoid the creation of new artificial radiometric information.

\subsubsection{Image matching}

Image matching is the most fundamental and also most challenging step in the reconstruction of a 3D surface based on 2D imagery. Initially, conjugate features or points need to be detected automatically in the overlapping images. The surface model can be processed afterwards by calculation of height differences, based on the measurement of the disparity between corresponding pixels. The accuracy, precision and completeness of the surface model are highly dependent on the performance of the image matching process.

An area-based matching algorithm is applied in the presented mixed sensor approach, comparing a patch of grey values on a reference image with patches on a search image. For the central pixel of the kernel on the reference image, the corresponding pixel in the search image needs to be detected, based on a moving window of certain pixel size and a mean normalized correlation coefficient. While the search window moves along the epipolar line, correlation with grey level distribution in the template patch is calculated for each position according to equation (5-8): 


$$
\rho=\frac{\sum_{i, j}\left[g_{1}\left(c_{1}, r_{1}\right)-\overline{g_{1}}\right]\left[g_{2}\left(c_{2}, r_{2}\right)-\overline{g_{2}}\right]}{\sqrt{\sum_{i, j}\left[g_{1}\left(c_{1}, r_{1}\right)-\overline{g_{1}}\right]^{2} \sum_{i, j}\left[g_{2}\left(c_{2}, r_{2}\right)-\overline{g_{2}}\right]^{2}}}
$$

with

$$
\begin{aligned}
& \overline{g_{1}}=\frac{1}{n} \sum_{i, j} g_{1}\left(c_{1}, r_{1}\right) \\
& \overline{g_{2}}=\frac{1}{n} \sum_{i, j} g_{2}\left(c_{2}, r_{2}\right)
\end{aligned}
$$

where $\rho$ is the correlation coefficient; $g(c, r)$ the grey value of the pixel $(c, r) ; c_{1}, r_{1}$ the pixel coordinates on the left image; $c_{2}, r_{2}$ the pixel coordinates on the right image; $n$ the total number of pixels within the moving window; and where $i, j$ refers to the pixel index within the moving window. A local maximum in the correlation signal indicates a possible matching candidate.

The applied algorithm works according to a coarse-to-fine hierarchical matching strategy. Image pyramids are used which consist of different versions of an image at exponentially decreasing resolutions, with the bottom level of the pyramid containing the original image. The matching results of each higher pyramid level are then used as approximations in each successive lower level. At each level an intermediate DSM is also generated from the matched points and refined through the image pyramid (Kallmann et al., 2003). Based on all data within each pyramid level, matching parameters are progressively determined and fine-tuned, following the method proposed by Kanade \& Okutomi (1994). This yields the adaptive selection of appropriate matching window size and shape, search distance and threshold for the image correlation value, based on the sort of imagery and the terrain type. The latter properties are measured via the use of noise modeling and image gradients.

In flat or homogenous regions, the matching window size should increase and the search distance should decrease. By contrast in rough areas or an urban environment, the patch should be smaller to prevent the matching window from extending discontinuities, while the search distance should be longer in order to deal with relief displacement. Due to the rough terrain type, here the template size was kept small with in most cases a value of seven by seven pixels. The 
search distance along epipolar lines was mostly set to a value of about 19 pixels, with the correlation threshold determined at 0.85 . This latter represents the minimum value necessary for a point to be accepted as a trustworthy match. In the case of correlation below the threshold, such matches cannot be trusted and are therefore removed from the point cloud. Although use of a larger correlation limit may result in greater accuracy, the number of successful matches will decrease, yielding a less dense point cloud and subsequent loss of detail. A smaller correlation limit, on the other hand, may increase the number of correlated points, but could also introduce a large amount of false matches.

Once corresponding points are matched and elevation parallax is measured, planimetric and altimetric ground coordinates for the projected object point can then be computed from the 2D image coordinates, according to 3D stereo forward intersection. With the availability of dense and accurate matching results, surface model extraction is a straightforward process. To create a connected and continuous terrain surface, the matched point cloud is interpolated to a regular $3 \mathrm{~m}$ spaced grid, a resolution providing a dense 3D description of the covered surface and also the best equilibrium between detail and reduction of noise. 


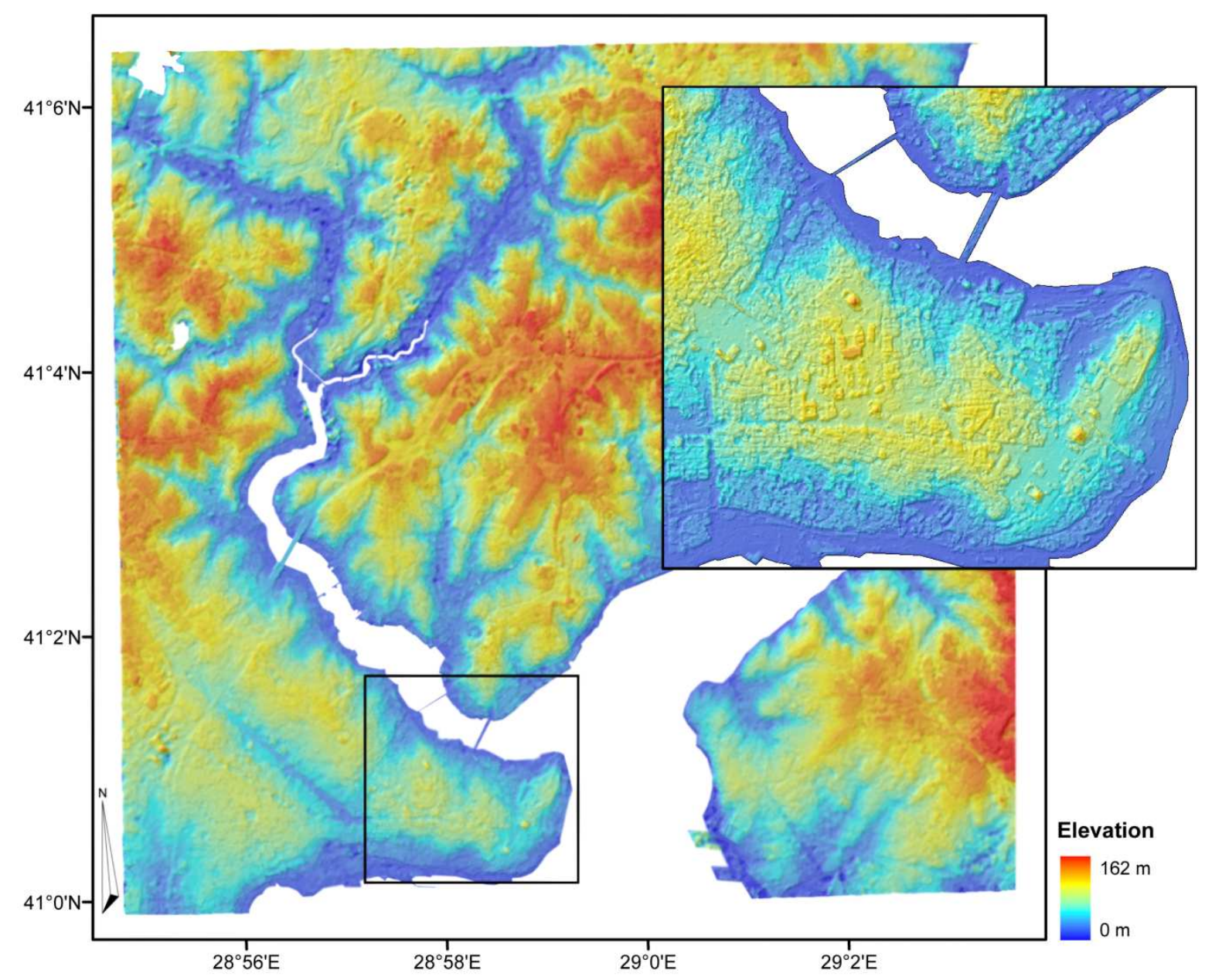

Figure 5-5. Map view on the 3 m color-coded DSM with enlargement of Istanbul's historic peninsula.

From a qualitative-visual perspective, the extracted DSM models terrain relief, topography and street-house patterns in a fairly veracious and detailed manner (see figure 5-5). Although various urban features are noticeable in detail, a certain degree of generalization is present. For example, the vast majority of close-set buildings are merged into larger blocks, as shadow, occlusion and divergences prohibit the detection and modeling of narrow streets in between. In addition, the adequacy of the spatial resolution and matching performance does affect DSM quality. Most of the time small dwellings, which are not captured by sufficient numbers of matched pixels, are either not reconstructed or manifest as a small spike in the surface model. Changes in plant phenology and in ground surface, due to construction works, result in erroneous parallax measurements and generate artifacts in the model. These sources of model error are discussed more thoroughly in section 5.4.3. Visual comparison with a reference DSM 
extracted from an Ikonos triplet reveals that less detail and more artifacts occur in the DSM extracted from different sensors.

Further DSM refinement via the use of techniques such as spatial filtering and shadow / occlusion removal is beyond the scope of this paper. The model is not postprocessed and thus only the accuracy of the 'raw' unfiltered DSM is assessed here.

\subsection{DIGITAL SURFACE MODEL ACCURACY ASSESSMENT}

The produced surface model is evaluated according to quantitative-statistical analysis involving comparison with three different types of reference data. In the first approach, the elevation model is assessed by comparison with 35 reference check points. The second approach compares the extracted DSM with a reference consisting of 3D rooftop building contours which are manually digitized on high resolution aerial images, while the third approach involves assessment of the overall accuracy of the generated model by comparison with a more accurate and more detailed surface model. Here a DSM extracted from an Ikonos triplet is used, since it has an adequate level of accuracy and detail. All three approaches have their own specific strengths and weaknesses, but can be considered complementary as they assess different aspects of the surface model.

The quality of reference points is expected to be much higher than that of rooftop contours or a reference DSM. The latter two were derived from remote sensing data, while the reference points were measured directly on the terrain and thus may be regarded as ground truth. On the other hand, the number of reference points is limited by both financial and logistical constraints. In addition they do not provide any information regarding building modeling quality. The reference model applied in the second approach is of high quality as the building contours were captured by well-trained operators, although the method exclusively assesses the modeling of building height. The third approach is carried out in order to provide an insight into the overall model accuracy for all land cover types.

This quantitative analysis and originating results only cover the overlapping area of the dataset with the respective references, since a suitable full coverage reference was not available. Due to the problematic nature of water bodies such 
as lakes, rivers and sea surface within the dataset, these homogeneous and lowtextured areas are manually digitized and masked out.

\subsubsection{Independent 3D reference points}

A dataset of 35 check points, distributed as uniformly as possible, is used to check the height accuracy. The check points are independent ground control points, which means that they are not used in the photogrammetric processing of the mixed sensor model. Residuals between accurately measured positions and those calculated by the model provide statistical information regarding the accuracy by which reality is modeled. The $\mathrm{Z}$ residuals are expressed in terms of an RMSE and are summarized in table 5-2.

Table 5-2. Geometric accuracy analysis based on check points.

\begin{tabular}{lcccc} 
& \multicolumn{4}{c}{ GPS check points } \\
\cline { 2 - 5 } & No. of GCP & RMSX & RMSY & RMSZ \\
\hline $\begin{array}{l}\text { A priori geometric } \\
\text { accuracy (m) }\end{array}$ & 14 & 0.81 & 0.87 & 2.43 \\
$\begin{array}{l}\text { DSM geometric } \\
\text { accuracy (m) }\end{array}$ & 35 & - & - & 2.96 \\
\hline
\end{tabular}

In table 5-2, first the a priori geometric accuracy is given, i.e. RMSE values for all the ground control points used to fix the mathematical relationship between image and object coordinate space. These values reflect the quality and robustness of the image orientation process. For X and Y, a sub-pixel level of accuracy is obtained, while the RMSE of the $\mathrm{Z}$ component is less than three pixels. The 35 independent check points are used to calculate the RMSZ error between measured positions and the values predicted by the model. Again RMSZ is less than three pixels.

\subsubsection{D building contour reference}

In the second approach, the mixed sensor model is investigated in terms of its ability to model buildings and urban structures. Only vertical positional accuracy of building roof surfaces is assessed. For two parts of the test site a reference database was made available by IMP-Bimtas, consisting of manually measured building heights. Both are densely built-up regions. They are illustrated in figure 
5-2 by a green dashed polygon. Zone UA1 covers $5.8 \mathrm{~km}^{2}$ and is located in the South of the test site. This area contains the city core, with the most prominent structures being blocks of connected multi-family townhouses and historic buildings. Zone UA2, located in the Southwest, covers $9.4 \mathrm{~km}^{2}$ and consists of an industrial area to the South and a new residential area to the North.

Rooftop heights are collected via the application of Z-map photo software on stereo aerial imagery involving digitization of the eaves. The aerial flight was conducted in July 2006 using an analogue JenOptik LC0030 camera (f= $305 \mathrm{~mm}$ ). Each image has a photo scale of 1:4500, a spatial resolution of $0.10 \mathrm{~m}$ and covers almost one $\mathrm{km}^{2}$. The reference dataset is assumed to be of much higher quality than the automatically generated DSM, because a skilled operator can extract elevation much more accurately than an automated approach. It is also assumed that the database of footprints is complete.

The assessment is also executed on an along-track Ikonos stereo pair, acquired in March 2002, which covers the same test site. The stereo constellation of the along-track pair is characterized by a convergence angle of $35.4^{\circ}$ or a corresponding base-to-height ratio of 0.67 . The surface model was extracted according to a similar methodology from radiometrically preprocessed images, based on 15 GCPs.

Firstly, difference maps visualizing the 3D error distribution are created by subtracting the 3D rooftop contours from the produced surface models according to a pixel-based approach. Secondly, statistical parameters are calculated only for values within the $95 \%$ confidence interval, based on these difference maps. The statistics are shown together in table 5-3, describing and summarizing the characteristics of the height differences between the generated surface models and the reference dataset. In total, 11.999 and 41.757 buildings are compared in zones UA1 and UA2, respectively.

Table 5-3. Descriptive statistics for building roof height calculated from difference elevation map of respectively "along-track Ikonos pair minus reference model and mixed sensor surface model minus reference" for $\mu \pm 2 \sigma$. 


\begin{tabular}{lcccc}
\cline { 2 - 4 } & \multicolumn{2}{c}{ Along-track pair } & \multicolumn{2}{c}{ Mixed sensor pair } \\
& Zone UA1 & Zone UA2 & Zone UA1 & Zone UA2 \\
\hline \# Compared pixels & 11999 & 41757 & 11999 & 41757 \\
Min dZ (m) & -11.51 & -12.89 & -7.52 & -7.06 \\
Max dZ (m) & 5.81 & 11.16 & 7.47 & 11.80 \\
Mean dZ (m) & -3.05 & -1.56 & -0.02 & 2.45 \\
St.dev. (m) & 3.39 & 4.56 & 3.81 & 4.81 \\
MAE (m) & 3.75 & 3.89 & 2.82 & 3.75 \\
RMSZ (m) & 4.56 & 4.82 & 3.81 & 5.40 \\
\hline
\end{tabular}

Some of the more significant quantitative measure values, illustrated in table $5-3$, should be clarified. As the reference is subtracted from the models, it can be stated that building heights in the along-track stereo pair model are underestimated. In the case of the mixed sensor model, there is a slight underestimation in zone UA1 and an overestimation of $2.45 \mathrm{~m}$ in zone UA2. The statistics in table 5-3 indicate that height differences are not spread out over a large range of values, but instead tend to be relatively close to the mean. The preciseness of the along-track pair is slightly better than that of the mixed sensor pair.

The Mean Absolute Error (MAE) parameter describes the central tendency of the data. It measures the average magnitude of errors without considering their direction. The MAE is a linear score, which means that all individual differences are weighted equally in the average. Remarkably, analysis of the MAE for both zones of the mixed sensor model reveals slightly smaller errors, despite the inherent problems of the model associated with temporal change and radiometric differences. As densely built-up areas are assessed, a small convergence angle performs better than a large one with regard to automatic image matching, even if images from different sensors are combined. The convergence angle of $17.2^{\circ}$ and base-to-height ratio of 0.4 of the multi-sensor pair therefore yields less building displacement and occlusion than the alongtrack Ikonos pair with its base-to-height ratio of 0.67.

As the errors are squared before being averaged, the RMSE assigns a relatively high weight to large errors, thus making the measure more sensitive to outliers. The mixed sensor approach performs better for the historic city centre area, but its value of RMSZ(95) for zone UA2 is $0.58 \mathrm{~m}$ higher than that of the along-track Ikonos pair. This is due to the higher frequency of large errors, to 
which a higher weight is assigned. Unlike the historic city centre in zone UA1 which has remained more or less unaltered, Zone UA2 is an area characterized by the occurrence of many changes, with such flux leading to mismatches between multi-temporal images. For the along-track pair, RMSZ(68) values are $3.83 \mathrm{~m}$ and $3.52 \mathrm{~m}$ for zones UA1 and UA2, respectively, while values of $2.02 \mathrm{~m}$ and $3.37 \mathrm{~m}$ are observed for the mixed sensor pair.

\subsubsection{DSM comparison between mixed sensor pair \& Ikonos triplet reference}

Vertical accuracy is collectively assessed for all types of land cover, by comparison of the mixed sensor surface model with a reference DSM (illustrated in figure $5-2$ by the yellow dotted polygon). The tri-stereoscopic approach, used to extract the reference surface model, yields highly accurate and detailed results. The redundancy of a third image results in more robust photogrammetric processing and matching, as well as in a reduction of distortions caused by occlusion, height discontinuities, etc. The surface model is extracted from radiometrically preprocessed images based on 15 GCPs collected using C-NAV DGPS. Assessment of the triplet DSM via comparison with 3D building vector data yields respective MAE(95) and RMSZ(95) values of $2.06 \mathrm{~m}$ and $2.60 \mathrm{~m}$ for zone UA1, and $2.44 \mathrm{~m}$ and $3.15 \mathrm{~m}$ for zone UA2. As the triplet surface model is considered a more optimal model, one can assume that it represents a better approximation of real terrain height. Large height differences between the triplet DSM and mixed sensor DSM can therefore be considered as errors in the mixed sensor surface model.

The altimetric accuracy is assessed according to a pixel-based comparison, with the reference dataset subtracted from the mixed sensor surface model. The resulting elevation difference map visualizes the 3D error distribution. The histogram of the elevation error map indicates a Gaussian distribution. Visual analysis of the map reveals a number of major divergences. As a next step in the mixed sensor approach, it was considered useful to investigate the significant sources of large elevation errors. Most are due to initial mistakes in point matching, consequently leading to erroneous parallax elevation measurements. In the worst case scenario, a correct match or solution simply does not exist. 
Either a wrong point is matched or no match established at all, leaving an empty height data value to be interpolated.

Analysis of distribution in the error map does not reveal any directional bias. The more noticeable large elevation differences are distributed randomly from a global perspective. Local mismatches around buildings are prominent due to occlusion and geometric dissimilarities caused by relief displacement, while other dense concentrations of differences can be detected in areas where many construction works have taken place. These areas occur mainly near the urban fringe in the North and West of the region. A final dense concentration of errors can be observed in the North, corresponding to a forested area.

Examples of areas in which large elevation differences occur are illustrated in figure 5-6. Figure 5-6(a), 5-6(b) and 5-6(c) show respectively a portion of the 2002 Quickbird ortho-image, the 2005 Ikonos ortho-image and the difference height map entitled 'mixed sensor surface model minus triplet reference model'. Analysis of these figures reveals the demolition of a building taking place during the three year time interval occurring between the acquisition of the two images. In figure 5-6(c), the model extracted from the multi-temporal images is compared with the reference DSM. Positive outliers are indicated by the deep red color, while negative outliers are shown in deep blue. Erroneous and poor matching in the changed area yields obviously large height errors. Observable in figure 5-6(a) and 5-6(b) are also the deformations of the changed area in the form of an image stretch or blurring, as the ortho-images are generated based on the badly modeled DSM. Figure 5-6(d), 5-6(e) and 5-6(f) show another error source that also can be categorized as 'no solution type'. Due to seasonal alterations in vegetation phenology, the radiometric response of each image differs significantly, thus making it impossible for a machine-based matching approach to detect homologous points. 

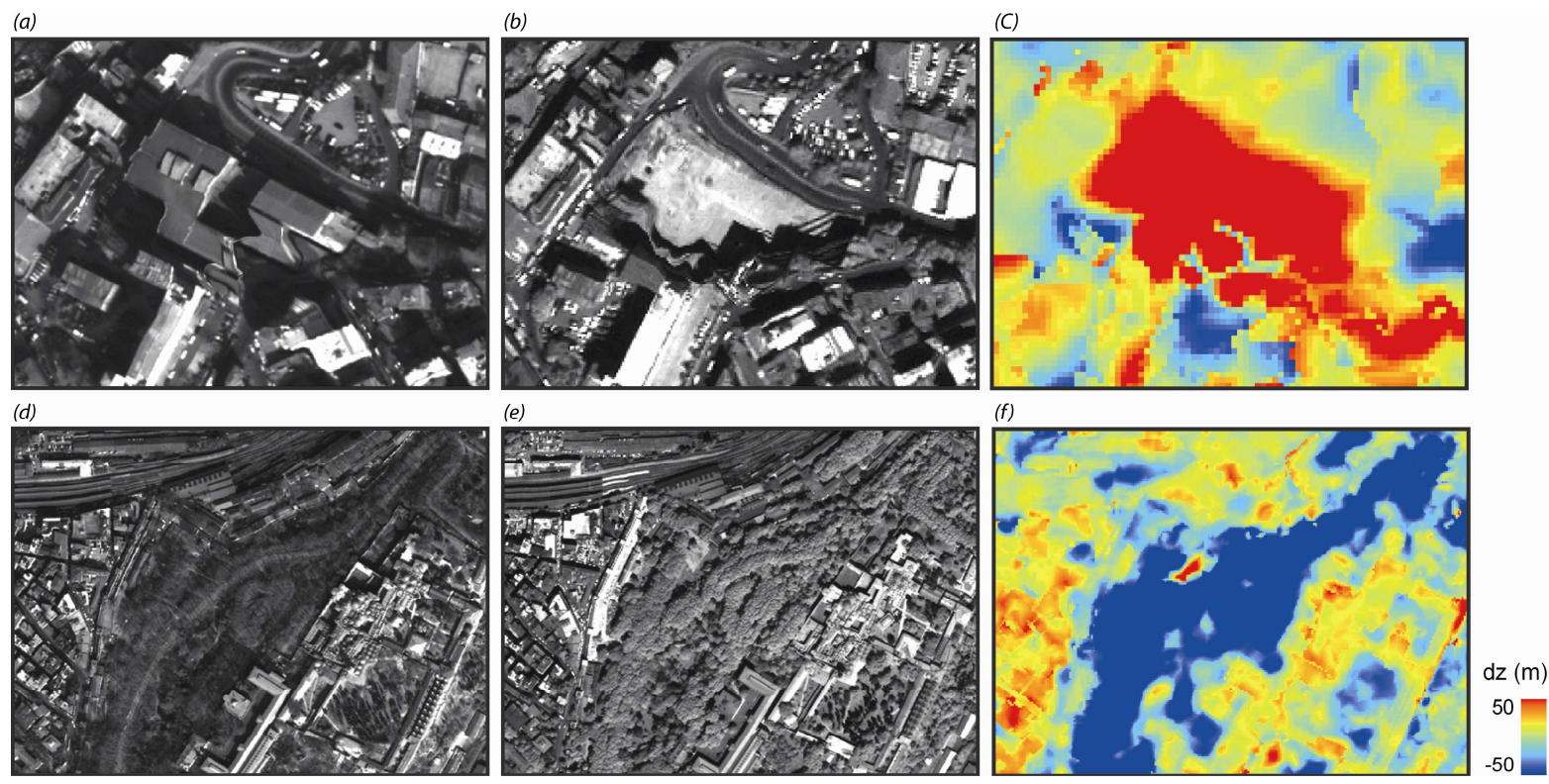

Figure 5-6. Elevation error sources in case of a mixed sensor approach. Two examples are illustrated where a correct matching solution does not exist.

Figure 5-6(a) and 5-6(d), 5-6(b) and 5-6(e), 5-6(c) and 5-6(f) are respectively a subimage from the Quickbird PAN image (2002-03-07), the Ikonos PAN image (2005-05-16) and the difference height map "mixed sensor surface model minus reference model". 5-6(a), 5-6(b) and 5-6(c) are an example of modeling errors due to ground surface changes between the image acquisitions. 5-6(d), 5-6(e) and 5-6(f) are an example of height errors due to changes in the phenological state of the vegetation.

In contrast to the 'no solution type' error sources mentioned above, there are other cases where a solution or high correlation match may exist, but which is non-trivial or not necessarily unique. Shadow is the most significant example of this error source type. Taken in March, the Quickbird image has a lower solar illumination angle and therefore shadow is more prominent. Error sources of this type can cause similar land cover or topographic features to appear to have changed in multi-date imagery, due to the different radiometric values held by the pixels. Radiometric enhancement and normalization can stretch and equalize the existing texture pattern in these areas, thus boosting the matching process. However, grey value dissimilarities still occur and enhancement is only able to reduce their effect.

Statistical parameters calculated from the difference map are presented in table 5-4, with the characteristics of the measured height differences between the generated and reference model described and summarized. This is carried out for the whole population, as well as for height differences within the $95 \%$ 
confidence interval after removal of outliers. The latter are usually caused by temporal topographic changes, vegetation, shadow, different illumination, etc.

Although the test site is a densely built-up area, approximately $21 \%$ is covered by vegetation, including parks, gardens and a forested area on the northern border of the urban fringe. As alterations in the phenological state of vegetation are a significant source of errors, a vegetation mask is generated based on LULC classification. Thereupon, pixels in the difference map which overlap with the vegetation mask are assigned a 'no data' value. In table 5-4, for the whole population as well as for the $95 \%$ confidence level, the statistical parameters are provided with and without the use of the vegetation mask. In total 7.619.098 pixels are compared without vegetation mask and 5.260.762 with the mask.

Table 5-4. Descriptive statistics calculated from difference elevation map "mixed sensor surface model minus reference model" for whole population and $\mu \pm 2 \sigma$.

\begin{tabular}{lcccc}
\cline { 2 - 5 } & \multicolumn{2}{c}{ Whole population } & \multicolumn{2}{c}{$\mathbf{9 5 \%}$ confidence level } \\
& Without & With veg. & Without & With veg. \\
& veg. mask & mask & veg. mask & mask \\
\hline \# Compared pixels & 7619098 & 5260762 & 7291122 & 5046475 \\
Min dZ (m) & -74.51 & -74.51 & -9.13 & -9.13 \\
Max dZ (m) & 128.81 & 128.81 & 13.99 & 12.40 \\
Mean dZ (m) & 2.46 & 1.98 & 2.15 & 1.68 \\
St.dev. (m) & 6.08 & 5.57 & 4.26 & 3.97 \\
MAE (m) & 4.20 & 3.76 & 3.59 & 3.21 \\
RMSZ (m) & 6.56 & 5.91 & 4.77 & 4.31 \\
\hline
\end{tabular}

Minimum and maximum height differences (i.e. errors) are significantly reduced after removal of the $5 \%$ outliers. For the remaining $95 \%$ of data, height differences range between a minimum of $-9.13 \mathrm{~m}$ and a maximum of $12.4 \mathrm{~m}$, with the attainable accuracy differing with land cover type. Flat, open terrain yields the smallest errors, whilst matching in (changed) vegetated areas is the most problematic, with an RMSZ of more than $8 \mathrm{~m}$ measured for the vegetation class areas of the test site. However, the use of a vegetation mask did not yield any significant improvement. The MAE(95) and RMSZ(95) are $3.21 \mathrm{~m}$ and $4.31 \mathrm{~m}$, respectively, while the RMSZ(68) with and without a vegetation mask is $2.89 \mathrm{~m}$ 
and $3.68 \mathrm{~m}$, respectively. These results are comparable with those obtained from the $3 \mathrm{D}$ building contour assessment.

\subsection{CONCLUSION \& DISCUSSION}

An approach has been discussed regarding the extraction of photogrammetric products from a mixed sensor stereo pair, acquired over an urban area. To the best of the authors' knowledge, as yet no research concerning DSM extraction over a densely built-up environment based on a high resolution mixed sensor stereo pair has been reported.

The three-dimensional information is extracted, solely based on remote sensing data and a set of GCPs, according to a highly automated matching algorithm. For cases where an along- or across-track pair from the same set of sensor imagery may not be available, are uneconomic or incompatible with required criteria, the methodology and results presented here demonstrate that a mixed sensor approach may offer a sound alternative. Results show that it is feasible to extract a DSM of a highly urbanized area from a mixed sensor pair, with accuracies comparable with those observed from a DSM extracted from an along-track pair. Hence, the flexibility of reconstructing valuable elevation models is greatly increased by considering the mixed sensor approach.

However, one should consider several criteria that must be taken into account during the image selection process, in order to construct a suitable stereo model. As discussed in section 5.2.2 the feasibility and performance of the method is strongly dependent on the given imagery and these criteria, with perhaps the most important being stereo constellation. Despite having a lower vertical accuracy (according to photogrammetric theory), it is advisable to select a smaller convergence angle when modeling an urban scene. This study demonstrates that similar, or even better, results can be achieved compared to DSM extraction from an along-track stereo pair, characterized by a larger convergence angle.

As the images, that constitute a mixed sensor pair, are acquired from a different orbital pass, at a different date or instant of time and by a different sensor system, radiometric and geometric dissimilarities can occur which hamper the image matching process. A radiometric enhancement and normalization of imagery is stressed to enhance image texture and to cope with 
the problems of different illumination and atmospheric conditions inherent in multi-temporal acquisitions. Epipolar resampling is mandatory in order to apply geometric normalization, or to remove image shifts in the Y-direction and differences in scale, rotation and spatial resolution.

The surface model extracted from the Ikonos-Quickbird pair is qualitatively and quantitatively assessed by comparison with different types of reference data. The comparison with reference points revealed the accuracy of the mixed sensor model to be within the theoretical boundaries claimed by the image vendors, i.e. $4 \mathrm{~m} \mathrm{CE90}$ and $5 \mathrm{~m}$ LE90 (GeoEye, 2006; DigitalGlobe, 2007). These values correspond to respective RMSE(95) values of $2 \mathrm{~m}$ in planimetry and $3 \mathrm{~m}$ in altimetry (Dial, 2000). The accuracies are above the theoretical values in the case of the two other quantitative assessment approaches, i.e. comparison with 3D building contours and with a DSM retrieved from an Ikonos triplet. This can be explained by the complexity of the test site since densely built-up areas usually yield lower accuracies. Even the along-track Ikonos pair yields a relatively low accuracy for this area. Secondly, the statistical analysis presented compares the mixed sensor model with reference surface models which are already biased, and not with ground truth, while the theoretical accuracies stated are for clearly identifiable, terrain-measured points (GeoEye, 2006; DigitalGlobe, 2007). Thirdly, CE90 and LE90 are used by the image vendors for communicating a theoretical accuracy, while the RMSE values report on the actually obtained accuracy of the model.

As the RFM sensor model is becoming the standard with which to determine the relationship between object space and the image space of pushbroom sensors, the described approach has a generic character and can be applied to other HRSI combinations without much adaptation. 


\section{ACKNOWLEDGEMENTS}

The Belgian Science Policy Office is gratefully acknowledged for funding the work presented in this paper (SR/00/105). Our sincerest thanks go both to the staff of the Remote Sensing Department of Istanbul Metropolitan Planning centre (IMPBimtas) for their broad support and hospitality, and to the other partners of the MAMUD project.

\section{REFERENCES}

Al-amri, A., Kalyankar, N.V., Khamitkar, S.D., 2010. Linear and Non-linear Contrast Enhancement Image. International Journal of Computer Science and Network Security, Vol. 10, N $^{\circ}$ 2, pp. 139-143.

Buyuksalih, G., Jacobsen, K., 2007. Digital Surface Models in Build Up Areas Based on Very High Resolution Space Images. Proc. ASPRS 2007 Annual Conference, Tampa, Florida, May 7-11, 2007, 8 p. (on CD-ROM).

Cain, J., 1989. Stereomodel Acquisition Geometry. PhD Dissertation, Technical Note 477, University of California, Berkeley, 270 p.

Crespi, M., Fratarcangeli, F., Giannone, F., Pieralice, F., 2009. Chapter 4 - Overview on Models for High Resolution Satellites Imagery Orientation. In: Li, D., Shan, J., Gong, J. (Eds.), Geospatial Technology for Earth Observation data. Springer, Heidelberg, pp. 63-104.

Devriendt, D., Goossens, R., Binard, M., 2006. Accuracy Assessment of an Ikonos Derived DSM over Urban and Suburban Area. Proc. EARSeL Symposium "New Developments and Challenges in Remote Sensing", Warsaw, Poland, May 29June 2, 2006, pp. 649-658.

Dial, G., 2000. Ikonos Satellite Mapping Accuracy. Proc. ASPRS 2000 Annual Conference, Washington D.C., May 22-26, 2000, 8 p. (on CD-ROM).

DigitalGlobe, 2007. Quickbird Imagery Products-Product Guide (Ver. 4.7.3). DigitalGlobe, USA, $77 \mathrm{p}$.

GeoEye, 2006. Ikonos-Imagery Products Guide (Ver. 1.5). GeoEye, USA, 19p.

Grodecki, J., 2001. Ikonos Stereo Feature Extraction - RPC Approach. Proc. ASPRS 2001 Annual Conference, St. Louis, Missouri, April 23-27, 2001, 7 p. (on CDROM). 
Grodecki, J., Dial, G., 2003. Block Adjustment of High-Resolution Satellite Images Described by Rational Functions. Photogrammetric Engineering and Remote Sensing, Vol. 69, Nㅜ 1, pp. 59-70.

Hall, F.G., Strebel, D.E., Nickeson, J.E., Goetz, S.J., 1991. Radiometric Rectification: Toward a Common Radiometric Response Among Multidate, Multisensor Images. Remote Sensing of Environment, Vol. 35, N1․ pp. 11-27.

Jacobsen, K., 2006. Digital Surface Models of City Areas by Very High Resolution Space Imagery. Proc. EARSeL Workshop of the SIG Urban Remote Sensing, Berlin, Germany, March 2-3, 2006, 10 p. (on CD-ROM).

Jazayeri, I., Fraser, C., 2008. Interest Operators in Close-Range Object Reconstruction. International Archives of Photogrammetry, Remote Sensing and Spatial Information Sciences, Vol. 37 (B5-1), pp. 69-74.

Kallmann, M., Bieri, H., Thalmann, D., 2003. Fully Dynamic Constrained Delaunay Triangulations. In: Brunnett, G., Hamann, B., Mueller, H. (Eds.), Geometric Modelling for Scientific Visualization. Springer, New York, pp. 241-258.

Kanade, T., Okutomi, M., 1994. A Stereo Matching Algorithm with an Adaptive Window: Theory and Experiment. Pattern Analysis and Machine Intelligence, Vol. 16, N 9, pp. 920-932.

Li, R., Zhou, F., Niu, X., Di, K., 2007. Integration of IKONOS and QuickBird Imagery for Geopositioning Accuracy Analysis. Photogrammetric Engineering and Remote Sensing, Vol. 73, № 9, pp. 1067-1074.

Morgan, M., Kim, K., Jeong, S., Habib, A., 2006. Epipolar Resampling of SpaceBorne Linear Array Scanner Scenes Using Parallel Projection. Photogrammetric Engineering \& Remote Sensing, Vol. 72, N 11, pp. 1255-1263. Poon, J., Fraser, C., Zhang, C., Zhang, L., Gruen, A., 2005. Quality Assessment of Digital Surface Models Generated from IKONOS Imagery. The Photogrammetric Record, Vol. 20, ํㅜㄴ. 110, pp. 162-171.

Raggam, H., 2006. Surface Mapping Using Image Triplets: Case Studies and Benefit Assessment in Comparison to Stereo Image Processing. Photogrammetric Engineering and Remote Sensing, Vol. 72, N 5, pp. 551-563.

Raggam, J., Almer, A., 1991. A Multi-Sensor Stereo-Mapping Experiment. Proc. ACSM-ASPRS-Auto Carto Annual Convention, Baltimore, Maryland, March 2528, 1991, pp. 173-182. 
Raggam, J., Almer, A., Strobl, D., 1992. Multisensor Mapping Using SAR in Conjunction with Optical Data. International Archives of Photogrammetry and Remote Sensing, Vol. 29 (B2), pp. 556-563.

Rozycki, S., Wolniewicz, W., 2007. Assessment of DSM Accuracy Obtained by High Resolution Stereo Images. Proc. ISPRS Hannover Workshop 2007 "High Resolution Earth Imaging for Geospatial Information", Leibniz, Germany, May 29 - June 01, 2007, 6 p. (on CD-ROM).

Shaker, A., 2008. Satellite Sensor Modeling and 3D Geopositioning Using Empirical Models. International Journal of Applied Earth Observation and geoinformation, Vol. 10, $\mathrm{N}^{\circ}$ 3, pp. 282-295.

Toutin, T., 1998. SPOT and Landsat Stereo Fusion for Data Extraction over Mountainous Area. Photogrammetric Engineering and Remote Sensing, Vol. 64, $\mathrm{N}^{\circ} 2$, pp. 109-113.

Toutin, T., 2001. Elevation Modeling from Satellite Visible and Infrared (VIR) Data. International Journal of Remote Sensing, Vol. 22, N 6, pp. 1097-1125.

Wallis, R., 1976. An Approach to the Space Variant Restoration and Enhancement of Images. Proc. Symposium on Current Mathematical Problems in Image Science, Monterey, California, 1976, pp. 641-662.

Welch, R., Jordan, R.T., Luvall, J.C., 1990. Geocoding and Stereo Display of Tropical Forest Multi-sensor Datasets. Photogrammetric Engineering and Remote Sensing, Vol. 56, N¹0, pp. 1389-1392.

Wolniewicz, W., Ke, L.C., 2006. Geometric Modeling of VHRS. Proc. International Calibration and Orientation ISPRS Workshop, Castelldefels, Spain, January 2527, 2006, 5 p. (on CD-ROM).

Zhang, L., Gruen, A., 2006. Multi-Image Matching for DSM Generation from Ikonos Imagery. ISPRS Journal of Photogrammetry and Remote Sensing, Vol. 60, $\mathrm{N}^{\circ} 3$, pp. 195-211.

Zhang, L., Pateraki, M., Baltsavias, E., 2002. Matching of Ikonos Stereo and Multitemporal GEO Images for DSM Generation. Proc. Map Asia, Bangkok, Thailand, August 7-9, 2002, 6 p. (on CD-ROM). 